\title{
Feasibility of Laparoscopic Surgery Assisted by a Robotic Telemanipulation System
}

\author{
J.P. Ruurda, I.A.M.J. Broeders, R.K.J. Simmermacher, I.H.M. Borel Rinkes, \\ and Th.J.M.V. van Vroonhoven \\ University Medical Center Utrecht, Department of Surgery, P.O. Box 85500, \\ 3508 GA Utrecht, the Netherlands \\ j.p.ruurda@chir.azu.nl
}

\begin{abstract}
Robotic telemanipulation systems have recently been introduced to enhance the surgeon's dexterity and visualisation in laparoscopic surgery. Technical feasibility of robot-assisted surgery was evaluated in 30 laparoscopic cholecystectomies.
\end{abstract}

\section{Introduction}

Where laparoscopic surgery offers the patient clear benefits, it introduces disadvantages to the surgeon as compared to open surgery. Manipulation is compromised by a limitation in degrees of freedom (DOF) of motion, inverted instrument response and variability in scaling, caused by working with long instruments through fixed entrypoints.

Visualisation is hampered by the indirect two-dimensional field of view. The hand-eye co-ordination is further deteriorated by the loss of the eye-hands-target axis, compromising normal oculovestibular input [1].

In order to cope with these limitations researchers started developing new tools for laparoscopic surgery, starting with camera guidance systems such as Aesop and Endoassist 23:4. Finally this resulted in the development of robotic telemanipulation systems. To demonstrate and evaluate technical feasibility of robotic assisted surgery, 30 laparoscopic cholecystectomies were performed.

\section{Methods}

30 robot-assisted laparoscopic cholecystectomies were performed with the Da Vinci system (Intuitive Surgical, Mountain View, California). The system consists of a master-console, and a 3 armed robotic telemanipulator, located at the operating table. From the console, the surgeon directly controls the camera-arm and the two instrument arms with two manipulators. The surgeon's motions are transposed to the tips of tiny instruments, where the Endowrist system provides the surgeon with seven DOF. The double optic system provides a 3D image, integrated in the console. The natural eye-hand-target axis is hereby restored.

Three surgeons were trained to perform laparoscopic cholecystectomies with the Da Vinci system. Set-up time, OR-time, complications and technical problems were recorded. 


\section{Results}

In 29 cases $(29 / 30 ; 97 \%)$ the cholecystectomy was completed laparoscopically. There was one conversion to an open procedure, caused by the surgeons' incapability to manipulate the gallbladder, due to severe cholecystitis. There were no robot-related complications. In three cases the replaceable blade of the electrocautery instrument detached, but could be removed during the same session.

The time needed to install the robotic system decreased with experience of the OR-crew. Operating time was comparable in robot-assisted cases to the time needed for laparoscopic cholecystectomy in the same clinic.

\section{Discussion}

Technical feasibility of robot assisted laparoscopic cholecystectomy was repeatedly demonstrated. No significant problems were noted during these procedures, except from the detachment of the electrocautery blade. The introduction of the second-generation blade solved this problem. The system showed to enhance the surgeon's dexterity and visualisation possibilities, providing intuitive control of the instruments.

Time-loss seems eventually to be overcome. Set-up and operating times will further decrease with increasing experience and further development of these robotic systems.

One of the points of criticism towards robotic systems, is the lack of tactile feedback. This is partly compensated for by the 3D visual feedback. Adding tactile feedback is one of the challenging topics in the development of these systems.

Although surgical robotics is considered to be in an early phase of development, the opportunities robotic telemanipulators offer are already distinct. They provide endoscopic surgeons with a dexterity incomparable to the way they used to perform laparoscopic surgery, allowing a precise and intuitive way of performing surgery.

\section{References}

1. Satava RM, Ellis SR. Human interface technology. An essential tool for the modern surgeon. Surg Endosc 1994; 8: 817-20

2. Sackier JM, Wang Y. Robotically assisted laparoscopic surgery. From concept to development. Surg Endosc 1994; 8: 63-6

3. Jacobs LK, Shayani V, Sackier JM. Determination of the learning curve of the AESOP robot. Surg Endosc 1997; 11: 54-5

4. Schurr M, Arezzo A, Buess GF. Robotics and systems technology for advanced endoscopic procedures: experiences in general surgery. Eur J Cardiothorac Surg 1999;16 Suppl 2: S97-105 